\title{
Quenching and propagation in KPP reaction-diffusion equations with a heat loss
}

\author{
Henri Berestycki* $\quad$ Francois Hamel $^{\dagger} \quad$ Alexander Kiselev $^{\ddagger} \quad$ Lenya Ryzhik $^{\S}$
}

April 13, 2004

\begin{abstract}
We consider a reaction-diffusion system of the KPP type in a shear flow and with a non-zero heat loss parameter. We establish criteria for the flame blow-off and propagation, and identify the propagation speed in terms of the exponential decay of the initial data. We prove existence of traveling fronts for all speeds $c>\max \left(0, c^{*}\right)$ in the case Le $=1$, where $c^{*} \in \mathbb{R}$. That seems to be one of the first non-perturbative results on the existence of fronts for the thermo-diffusive system in higher dimensions.
\end{abstract}

\section{Introduction}

There has been a lot of interest recently in the effect of flows on the qualitative and quantitative behavior of solutions of reaction-diffusion equations, such as existence of travelling fronts [5, 6, 8, 23, $25,26,27,28]$, speed-up of propagation [3, 4, 10, 14, 18, 21, 29], and flame quenching [11, 24], see $[8,28]$ for recent reviews of the mathematical results in the area. This problem has also attracted a significant attention in the physical literature, we mention $[1,2,15,16,17]$ among the recent papers and refer to [22] as a general reference. However, most of the rigorous mathematical analysis have dealt so far almost exclusively with the flow effect for a single reaction-diffusion equation, or for a system close to a scalar equation with the Lewis number close to unity [7].

The purpose of this paper is to analyze the behavior of the solutions of a reaction-diffusion system of KPP type

$$
\begin{aligned}
& \frac{\partial T}{\partial t}+A u(y) \frac{\partial T}{\partial x}=\Delta T+T Y \\
& \frac{\partial Y}{\partial t}+A u(y) \frac{\partial Y}{\partial x}=\frac{1}{\mathrm{Le}} \Delta Y-T Y
\end{aligned}
$$

with a non-trivial shear flow $u(y)$. Here $T$ is the temperature of the reactant, $Y$ its concentration, and the parameter $A$ measures the flow amplitude. The Lewis number Le is the ratio of the thermal and material diffusivities.

We assume that the initial profiles $T_{0}$ and $Y_{0}$ are bounded and satisfy

$$
T_{0}=O\left(e^{-\lambda x}\right), \quad Y_{0} \rightarrow 1 \text { as } x \rightarrow+\infty
$$

*EHESS, CAMS, 54 Boulevard Raspail, F-75006, Paris, France; hb@ehess.fr

${ }^{\dagger}$ Université Aix-Marseille III, LATP, Faculté Saint-Jérôme, Case Cour A, Avenue Escadrille Normandie-Niemen, F-13397 Marseille Cedex 20, France; francois.hamel@univ.u-3mrs.fr

${ }^{\ddagger}$ Department of Mathematics, University of Wisconsin, Madison, WI 53706, USA; kiselev@math.wisc.edu

$\S$ Department of Mathematics, University of Chicago, Chicago, IL 60637, USA; ryzhik@math.uchicago.edu 
so that it is physically reasonable to expect that solution would propagate to the right where the fresh mixture is available for chemical reaction.

The system (1.1) is considered in a cylindrical domain $D=\mathbb{R}_{x} \times \Omega_{y}$, with the heat-loss boundary conditions:

$$
\frac{\partial T}{\partial n}+q T=0, \quad \frac{\partial Y}{\partial n}=0, \quad y \in \partial \Omega .
$$

Here $q \geq 0$ is the heat loss parameter, and $\Omega \in \mathbb{R}^{N-1}$ is a smooth bounded domain with the outward unit normal denoted by $n$. The flow $u(y)$ is continuous in $\bar{\Omega}$ with mean zero:

$$
\int_{\Omega} u(y) d y=0
$$

The situation when the mean flow is not zero can be reduced to this case by a change of coordinates.

In the particular case when the heat-loss parameter $q=0$, Le $=1$ and we have initially $T_{0}+Y_{0}=$ 1, the system (1.1) reduces to a single KPP equation

$$
\frac{\partial T}{\partial t}+A u(y) \frac{\partial T}{\partial x}=\Delta T+T(1-T)
$$

It is well known [20] that in this case, provided that $T_{0} \sim e^{-\lambda x}$ as $x \rightarrow+\infty$ and

$$
\liminf _{x \rightarrow-\infty, y \in \bar{\Omega}} T_{0}(x, y)>0,
$$

solution propagates with the speed $c[\lambda]$. The latter is determined by the decay rate $\lambda$ of the initial data as follows. Let $\mu_{N}(\lambda)$ be the first eigenvalue of the following problem in $\Omega$

$$
-\Delta \psi_{N}-A \lambda u(y) \psi_{N}=\mu_{N}(\lambda) \psi_{N}, \quad y \in \Omega
$$

with the Neumann boundary condition

$$
\frac{\partial \psi_{N}}{\partial n}=0, \quad y \in \partial \Omega
$$

Then the speed $c$ is determined by

$$
c \lambda-\lambda^{2}=1-\mu_{N}(\lambda)
$$

Furthermore, as $\mu_{N}(\lambda)$ is a concave function [8], if one views (1.8) as an equation for $\lambda$, solutions exist for all $c \geq c^{*}$, where $c^{*}$ is a threshold parameter. It has been shown in [8] that whatever the fluid flow $u(y)$ is and for all $A$, travelling front solutions of (1.5) also exist if and only if $c \geq c^{*}(A, u)$. Solutions with the initial data that decay faster than the critical exponential rate $\lambda_{*}$, propagate with the minimal speed $c^{*}$.

We show in this paper that the presence of heat-loss, that is, a non-zero heat-loss parameter $q>0$ in the boundary conditions (1.3), changes the qualitative behavior of solutions of (1.1). Depending on the shear flow profile $u(y)$ and its amplitude $A$, solution with a given initial data may either propagate, become extinct or be blown-off. We say that the flame becomes extinct if $\|T(t)\|_{L^{\infty}} \rightarrow 0$ as $t \rightarrow+\infty$. The flame is blown-off if there exists a function $\Phi(\xi)$ so that $\Phi(\xi) \rightarrow 0$ as $\xi \rightarrow+\infty$, and $T(t, x, y) \leq \Phi(x+c t)$ with $c>0$. Note that the extinction is a stronger property than blow-off. We will see that there are situations where the flame is blown off but does not become extinct. 
Qualitatively our results are as follows. First, provided that the initial data satisfies (1.2), the behavior of the solution is completely determined by the decay rate $\lambda$ and the leading eigenvalue $\mu(\lambda)$ of problem (1.6) with the heat loss (Robin type) boundary conditions

$$
\begin{array}{r}
-\Delta \phi-A \lambda u(y) \phi=\mu(\lambda) \phi, \quad y \in \Omega \\
\frac{\partial \phi}{\partial n}+q \phi=0, \quad y \in \partial \Omega .
\end{array}
$$

First, we will show that if $\mu(0)>1$ then solution becomes extinct for large times. Furthermore, under the general condition that there exists $\lambda_{0}>0$ so that $\mu\left(\lambda_{0}\right)>1$ we show that a sufficiently strong advection $A>A_{0}(\lambda)$ blows the flame off. Otherwise, if none of the above occur, solution to (1.1) propagates to the right with the speed $c(\lambda)$ determined by (1.8) with $\mu_{N}$ replaced by $\mu(\lambda)$. Note that the speed $c(\lambda)$ is independent of the Lewis number. This is a special feature of the KPP type systems that does not generalize to other types of nonlinearities. We should mention that Billingham and Needham [9] showed that the minimal speed for (1.1) is independent of the Lewis number, but in the absence of flow or heat loss and in one dimension. A uniform upper bound for the burning rate that is independent of the Lewis number in the presence of a periodic flow but with no heat loss has been obtained in [19]. Finally, we show that a pulse shape travelling front exists when Le $=1$ and the heat loss parameter satisfies $q>0$. We emphasize that this is still a genuine system despite the assumption Le $=1$ since (1.1) does not reduce to a single equation as long as $q>0$. Our results agree qualitatively with the numerical studies of the heat-loss effect for systems of reaction-diffusion equations, albeit with the Arrhenius nonlinearity, in $[12,13]$. Our method applies only to the KPP type of reaction term and treatment of other reaction types remains an open problem.

This paper is organized as follows. Front blow-off and extinction are discussed in Section 2. The results on the asymptotic speed of propagation are presented in Section 3, while Section 4 is devoted to the existence of travelling waves and their qualitative properties.

Acknowledgement. AK and LR were supported by NSF grants DMS-0321952 and DMS0314129, and DMS-0203537, and by Alfred P. Sloan fellowships. They also are grateful to the program "Dynamique des fronts réactifs" at Institut Henri Poincaré, where this work has been initiated, for hospitality.

\section{Flame extinction and blow-off}

We investigate in this section the possibility of blow-off or extinction of solutions of the reactiondiffusion system (1.1). As mentioned in the introduction, this system is considered in a domain $D=\mathbb{R}_{x} \times \Omega_{y}$, with the heat-loss boundary conditions (1.3). We assume that the initial data $T_{0}, Y_{0}$ is non-negative, satisfies $0 \leq T_{0}, Y_{0} \leq 1$, and, moreover, $T_{0}(x, y) \leq C e^{-\lambda x}$ with some $\lambda>0$. The flow $u(y)$ is continuous in $\bar{\Omega}$ with mean zero (1.4).

Let us denote by $\mu_{s}$ and $\phi_{s}(y)$ the principal eigenvalue and eigenfunction, respectively, of the following problem defined in the cross-section $\Omega$ with Robin boundary conditions:

$$
\begin{aligned}
& -\Delta_{y} \phi_{s}-s u(y) \phi_{s}=\mu_{q}(s) \phi_{s} \text { in } \Omega, \\
& \frac{\partial \phi_{s}}{\partial n}+q \phi_{s}=0, \quad y \text { on } \partial \Omega .
\end{aligned}
$$

The eigenfunction $\phi_{s}(y)$ is positive, and is normalized so that

$$
\int_{\Omega} \phi_{s}^{2}(y) d y=1
$$


Moreover, with this normalization one gets

$$
\mu_{q}(s)=\int_{\Omega}\left|\nabla \phi_{s}\right|^{2} d y+q \int_{\partial \Omega} \phi_{s}^{2} d S_{y}-s \int_{\Omega} u(y) \phi_{s}^{2}(y) d y .
$$

In particular, for any $q>0$ we have $\mu_{q}(0)>0$ in contrast with the adiabatic case $q=0$, where $\mu_{0}(0)=0$. The variational principle

$$
\mu_{q}(s)=\inf _{\psi \in H^{1}(\Omega),\|\psi\|_{2}=1}\left(\int_{\Omega}|\nabla \psi|^{2} d y+q \int_{\partial \Omega} \psi^{2} d S_{y}-s \int_{\Omega} u(y) \psi^{2}(y) d y\right)
$$

implies that the function $\mu_{q}(s)$ is concave as an infimum of a family of affine functions. This may also be seen by a formal differentiation (which can be made rigorous) with respect to $s$. Indeed, differentiating (2.1) with respect to $s$ we obtain

$$
-\Delta \phi_{s}^{\prime}-s u(y) \phi_{s}^{\prime}-u(y) \phi_{s}=\mu_{q}^{\prime}(s) \phi_{s}+\mu_{q}(s) \phi_{s}^{\prime},
$$

where the prime denotes derivative with respect to $s$. We multiply (2.1) by $\phi_{s}^{\prime}$, and (2.4) by $\phi_{s}$, integrate and subtract one equation from the other. Using the fact that

$$
\int_{\Omega} \phi_{s}(y) \phi_{s}^{\prime}(y) d y=0
$$

and the $L^{2}$-normalization of $\phi_{s}$, we obtain

$$
\mu_{q}^{\prime}(s)=\frac{d \mu_{q}}{d s}(s)=-\int_{\Omega} u(y) \phi_{s}^{2}(y) d y
$$

so that

$$
\frac{d^{2} \mu_{q}}{d s^{2}}(s)=-2 \int_{\Omega} u(y) \phi_{s}(y) \phi_{s}^{\prime}(y) d y .
$$

Multiplying (2.4) by $\phi_{s}^{\prime}$ and integrating we obtain using the above expressions

$$
q \int_{\partial \Omega}\left(\phi_{s}^{\prime}\right)^{2} d S_{y}+\int_{\Omega}\left|\nabla \phi_{s}\right|^{2} d y-s \int_{\Omega} u(y)\left(\phi_{s}^{\prime}\right)^{2} d y+\frac{1}{2} \frac{d^{2} \mu_{q}}{d s^{2}}=\mu_{s} \int_{\Omega}\left(\phi_{s}^{\prime}\right)^{2} d y .
$$

The variational principle $(2.3)$, with $\psi=d \phi_{s} / d s$ used as a test function, implies then that $\frac{d^{2} \mu_{q}}{d s^{2}}(s) \leq$ 0 so that $\mu_{q}$ is concave.

The following result shows that extinction and blow-off are controlled by the behavior of $\mu_{q}(s)$.

Theorem 1 1. Blow-off. Let us assume that there exists $s_{0}$ so that $\mu_{q}\left(s_{0}\right)>1$. Then there exists $A_{0} \in\left[0, \max \left(s_{0} / \lambda, s_{0} / \sqrt{\mu_{q}\left(s_{0}\right)-1}\right)\right]$ so that for all $A>A_{0}$ we have $T(t, x, y) \leq C e^{-\eta(x+\gamma t)}$ for all $t \geq 0$. The positive constants $\eta$ and $\gamma$ depend on $A$, and there exists a constant $C>0$ so that the blow-off speed satisfies $\gamma(A) \geq C A$ for all $A \geq A_{0}$.

2. Extinction. If $\mu_{q}(0)>1$, then $T(t, x, y) \leq C e^{-\gamma_{0} t}$ for all $A$ with $\gamma_{0}>0$ independent of $A$.

Remark. A natural question is whether the blow-off described in the first part of the theorem is different from complete extinction. In the discussion after the proof, we show that if $\mu_{q}(0)<1$, then we have blow-off but not extinction for appropriate initial data. 
Proof. We observe first that $0 \leq Y(t, x, y) \leq 1$ for all $t>0$ as follows from the maximum principle. Therefore $0 \leq T(t, x, y) \leq \psi(t, x, y)$, where $\psi$ is any solution of

$$
\begin{aligned}
& \frac{\partial \psi}{\partial t}+A u(y) \frac{\partial \psi}{\partial x} \geq \Delta \psi+\psi, \quad(x, y) \in \Omega \\
& \frac{\partial \psi}{\partial n}+q \psi=0, \quad y \in \partial \Omega
\end{aligned}
$$

provided that $T_{0}(x, y) \leq \psi(0, x, y)$. Let us look for the super-solution $\psi$ in the form $\psi(t, x, y)=$ $C e^{-\eta(x+\gamma t)} \phi_{s}(y)$ with $s=\eta A$. Insert this expression into (2.7) and obtain that we need

$$
-\eta \gamma \phi_{s}-\eta A u(y) \phi_{s} \geq \eta^{2} \phi_{s}+\Delta_{y} \phi_{s}+\phi_{s} .
$$

This is true provided that

$$
\eta \gamma \leq \mu_{q}(\eta A)-1-\eta^{2}
$$

Therefore such a super-solution exists if we may find a constant $\eta$ such that $\mu_{q}(\eta A)-1-\eta^{2}>0$ and $0 \leq \eta \leq \lambda$. This indeed holds for $\eta=s_{0} / A$ as long as $A>A_{0}=\max \left(s_{0} / \lambda, s_{0} / \sqrt{\mu_{q}\left(s_{0}\right)-1}\right)$. The blow-off speed $\gamma$ then may be chosen as

$$
\gamma=\frac{A}{s_{0}}\left(\mu_{q}\left(s_{0}\right)-1-\frac{s_{0}^{2}}{A^{2}}\right) .
$$

This proves the first part of the theorem.

The second (extinction) part of Theorem 1 is proved similarly: if $\mu_{q}(0)>1$ then the function $\psi(t, y)=C e^{-\left(\mu_{q}(0)-1\right) t} \phi_{0}(y)$ is a super-solution of (2.7) which proves the second statement in the theorem. Notice that for this part of the theorem, it is not necessary to assume that $T_{0}$ decays as $x \rightarrow+\infty$.

An interesting observation is that it is possible that in the absence of the flow the front will propagate, while a sufficiently strong flow will blow it off. This happens for flows $u(y)$ and heat-loss parameters $q>0$ such that $\mu_{q}(0)<1$ while there exists $s_{0}>0$ so that $\mu_{q}\left(s_{0}\right)>1$. An example of such a flow may be constructed as follows. We observe that at $s=0$ fixed we have $\mu_{q}(0) \rightarrow \mu_{D}$, as $q \rightarrow+\infty$, where $\mu_{D}$ is the first Dirichlet eigenvalue for the domain $\Omega$. Let us choose a domain $\Omega$ so that $\mu_{D}>1$ so that there exists a heat-loss parameter $q_{0}>0$ such that $\mu_{q_{0}}(0)=1$, as $\mu_{q_{0}}(0)$ is a continuous increasing function of $q$ (as follows from $(2.3)$ ) and $\mu_{0}(0)=0$. We also choose a mean-zero flow $u(y)$ so that

$$
\frac{d \mu_{q}}{d s}(0)=-\int_{\Omega} u(y) \phi_{0}^{2}\left(y ; q_{0}\right) d y>0 .
$$

This is possible since $\phi_{0}\left(y ; q_{0}\right)$ is not constant and is independent of the flow $u(y)$. Then there exists $s_{0}>0$ so that $\mu_{q_{0}}\left(s_{0}\right)>1$. Continuity and monotonicity of $\mu_{q}(s)$ as a function of $q$ imply that there exists $q_{1}<q_{0}$ so that $\mu_{q_{1}}(0)<1$ while $\mu_{q_{1}}\left(s_{0}\right)>1$ so that a flame with a heat-loss parameter $q_{1}$ propagates when the flow is absent (we will see below in Section 3 that condition $\mu_{q}(0)<1$ is sufficient for propagation at $A=0$ ), but is blown-off when the flow amplitude $A$ is sufficiently large.

Condition (2.9) is quite natural for a blow-off. Let us explain roughly the intuition behind it. Due to the heat loss, the function $\phi_{0}\left(y ; q_{0}\right)$ is smaller near the boundary of $\Omega$. Therefore, in order to satisfy both (2.9) and the mean-zero assumption (1.4) the flow $u$ should be directed against the flame in the middle of the cross-section $\Omega$ and push the flame forward near the walls. However, the flame is cooled by the heat-losses near the boundary balancing the push forward effect of the flow so that the counter-flow effect in the middle dominates and the flame is blown-off. This scenario agrees with the numerical observations of $[12,13]$ for the Arrhenius nonlinearity. 
We remark that the blow-off described in the first part of the theorem, in general, does not lead to extinction, and so is a different phenomenon. Indeed, denote by $\tilde{\mu}_{q}(s)$ the eigenvalue defined by (2.3) with the opposite sign of the advection term, that is, with $u(y)$ replaced by $-u(y)$. We have $\mu_{q}(0)<1$ and $\mu_{q}\left(s_{0}\right)>1$, which implies that $d \mu_{q}(0) / d s(0)>0$ because of the concavity property. But $d \tilde{\mu}_{q} / d s(0)=-d \mu_{q} / d s(0)$, and due to the concavity $\tilde{\mu}_{q}(s)<1$ for all $s>0$. Assume that the initial data $T_{0}, Y_{0}$ satisfy appropriate bounds (see (3.13), (3.14)) for $x \leq 0$ as well as for $x \geq 0$. Then our results on the propagation of fronts which appear in the following section (Theorem 3 ) show that a front propagating to the left will form, and thus there will be no complete extinction.

Note that in the adiabatic case the blow-off effect is impossible. Indeed, when $q=0$, the function $s \mapsto \mu_{q}(s)$ is strictly decreasing if $u$ satisfies (1.4) and $u \not \equiv 0$. Indeed, $\mu_{0}(0)=0$ and $d \mu_{0} / d s(0)=0$ as implied by $(2.6)$ and the fact that $\phi_{0}(y)=1$ in the adiabatic case. Then concavity of $\mu_{0}(s)$ implies that $\mu_{0}(s)$ is indeed decreasing for $s \geq 0$ in the case $q=0$ (notice that $\phi_{s}$ is not constant for $s>0$, since $u \neq \equiv 0$ ) so that $\mu_{0}(s)<1$ for all $s>0$ and the flame blow-off is impossible (see the results of the following section). One might expect this from physical considerations, as well as from a universal positive lower bound on the burning rate obtained in [10] in the case Le $=1$.

Finally, we observe that the critical blow-off amplitude $A_{0}$ depends on the exponential decay rate $\lambda$ of the initial data: $A_{0} \sim s_{0} / \lambda$. It will be clear from our consideration of flame propagation in the next section that it is indeed possible that at a fixed flow amplitude $A$ the initial data with, say, compact support is blown off while sufficiently slowly decaying fronts will propagate.

\section{$3 \quad$ Flame propagation}

We present in this section results on flame propagation and identify the speed of the flame in terms of the rate of exponential decay of initial data. It is well known that the asymptotic speed of propagation for a solution of a single KPP equation is determined by the rate of decay of the initial data. This result has been generalized to a single KPP equation in a shear flow in [20]. We present in this section a version of such a result for a reaction-diffusion system with an arbitrary Lewis number and a non-zero heat loss parameter. The tools of analysis here are quite different from previous works as we have to deal with a genuine system case that lacks a maximum principle. In particular sub- and supersolutions techniques do not readily apply, and new ingredients are required. The idea of the proof still involves finding sub- and supersolutions for temperature and concentration that move with the same speed, but a new way of constructing them is required. They are constructed by iteration, starting with a super-solution $\bar{Y}_{1}=1$ for the concentration, that itself is in turn used

to produce a super-solution $\bar{T}_{1}$ of temperature, that is used to obtain a sub-solution $\underline{Y}_{1}$, which leads to a sub-solution $\underline{T}_{1}$. This process may be continued leading to improving upper and lower bounds for the temperature and concentration but the key point is that, at the end of the day, the sub- and super-solutions propagate with the same speed.

\subsection{Flame propagation in a uniform medium}

We consider first for simplicity the case when the flow is absent: $u(y) \equiv 0$, and temperature $T$ and concentration $Y$ satisfy the KPP system

$$
\begin{aligned}
& \frac{\partial T}{\partial t}=\Delta T+T Y \\
& \frac{\partial Y}{\partial t}=\frac{1}{\mathrm{Le}} \Delta Y-T Y
\end{aligned}
$$


with the boundary conditions (1.3) and with an arbitrary Lewis number Le $>0$. Let us make precise the assumptions on the asymptotic behavior of the initial conditions:

$$
\begin{aligned}
& 0 \leq T_{0}, \quad T_{0} \text { is bounded, } \quad 0 \leq Y_{0} \leq 1 \\
& C_{1} e^{-\lambda x} \leq T_{0}(x, y) \leq C_{2} e^{-\lambda x}, \quad x \geq 0, y \in \bar{\Omega} \\
& 1-Y_{0}(x, y) \leq C_{3} e^{-\lambda^{\prime} x}, \quad x \geq 0, y \in \bar{\Omega}, \quad \lambda^{\prime}>0
\end{aligned}
$$

for some positive constants $C_{1}, C_{2}, C_{3}$ and $\lambda^{\prime}$. In the following, let $\phi(y)$ be the positive eigenfunction of the Laplacian with the Robin boundary condition:

$$
\begin{aligned}
& -\Delta \phi=\mu \phi, \quad y \in \Omega \\
& \frac{\partial \phi}{\partial n}+q \phi=0, \quad y \in \partial \Omega .
\end{aligned}
$$

With the notation of (2.1), we have $\mu=\mu_{q}(0)$ and $\phi=\phi_{0}$ (up to a normalization of $\phi$ ). We know that $\mu>0$. We assume here that $\mu<1$ so that quenching does not happen and we choose the speed $c$ depending on the rate $\lambda$ so that

$$
c \lambda-\lambda^{2}=1-\mu .
$$

We will assume that $0<\lambda<\lambda_{*}=\sqrt{1-\mu}$ and thus $c>c_{*}=2 \sqrt{1-\mu}$. We have the following theorem.

Theorem 2 Let us assume that $\mu<1$ and $0<\lambda<\sqrt{1-\mu}$, then the solution of (3.1-3.2) moves with speed $c$ in the following sense: for any $c^{\prime}>c$ and any $(x, y) \in \mathbb{R} \times \bar{\Omega}$ we have $T\left(t, x+c^{\prime} t, y\right) \rightarrow 0$ as $t \rightarrow+\infty$, while for the speed $c$ itself, one can find $x_{0} \in \mathbb{R}$ and $\alpha\left(x_{0}, y\right)>0$ such that $T\left(t, x_{0}+c t, y\right) \geq$ $\alpha\left(x_{0}, y\right)$ for all $t \geq 1$ and $y \in \Omega$. Moreover, there exists a nonnegative, not identically zero function $G(x, y)$ such that if $T_{0}(x, y) \geq G(x, y)$, then $T(t, x+c t, y) \geq G(x, y)$ for all $t$.

Proof. As explained in the beginning of this section we will construct sub- and super-solutions for $T(t, x, y)$ that both move with speed $c$. Recall that $0 \leq T(t, x, y)$ and $0 \leq Y(t, x, y) \leq 1$ according to the maximum principle. Thus, the function

$$
\bar{T}(t, x, y)=M e^{-\lambda(x-c t)} \phi(y)
$$

satisfies the Robin boundary conditions $\frac{\partial \bar{T}}{\partial n}+q \bar{T}=0$ on $\partial \Omega$ and

$$
\frac{\partial \bar{T}}{\partial t}=\Delta \bar{T}+\bar{T} \geq \Delta \bar{T}+\bar{T} Y
$$

for all $M>0$, as follows from the definition of $c$. Therefore, we have $T(t, x, y) \leq \bar{T}(t, x, y)$ when $M$ is large enough, because such an inequality holds at $t=0$.

We then have the exponential bound

$$
T(t, x, y) \leq M e^{-\lambda(x-c t)} \phi(y) .
$$

This implies in particular that for $\left(x_{0}, y\right) \in \mathbb{R} \times \bar{\Omega}$ and $c^{\prime}>c$ we have

$$
T\left(t, x_{0}+c^{\prime} t, y\right) \leq M e^{-\lambda\left(x_{0}+c^{\prime} t-c t\right)} \rightarrow 0 \text { as } t \rightarrow+\infty
$$

which proves the first part of the theorem. 
Let us briefly consider the special case Le $=1$. Here, assuming that $T_{0}+Y_{0} \leq 1$, we have, in addition to (3.4), that $T(t, x, y) \leq 1$. This is because the function $Z=T+Y$ then satisfies

$$
\begin{aligned}
& \frac{\partial Z}{\partial t}=\Delta Z \text { in } \Omega \\
& \frac{\partial Z}{\partial n}=-q T \leq 0, \quad y \text { on } \partial \Omega
\end{aligned}
$$

so that $Z(t, x, y) \leq \sup \left(T_{0}+Y_{0}\right) \leq 1$. Therefore, $T$ satisfies

$$
\begin{aligned}
& \frac{\partial T}{\partial t} \leq \Delta T+T(1-T) \text { in } \Omega \\
& \frac{\partial T}{\partial n}+q T=0, \quad y \text { on } \partial \Omega
\end{aligned}
$$

so that indeed $T \leq 1$. Therefore, if Le $=1, T(t, x, y) \leq U(x-c t)$, with $U(\xi)=\min \left\{1, M e^{-\lambda \xi}\right\}$ provided we choose $T_{0}$ such that

$$
T_{0}(x, y) \leq \frac{M e^{-\lambda x} \phi(y)}{\|\phi\|_{\infty}} .
$$

The special properties available for Le $=1$ case will be important in the Section 4 , where we turn to the construction of travelling fronts.

Coming back to the general case Le $>0$, the previous consideration and the maximum principle imply that $Y(t, x, y) \geq \tilde{Y}(t, x, y)$, where the function $\tilde{Y}$ solves

$$
\begin{aligned}
& \frac{\partial \tilde{Y}}{\partial t}=\frac{1}{\mathrm{Le}} \Delta \tilde{Y}-M e^{-\lambda(x-c t)} \tilde{Y} \\
& \frac{\partial \tilde{Y}}{\partial n}=0, \quad y \in \partial \Omega \\
& \tilde{Y}(0, x, y)=Y_{0}(x, y) .
\end{aligned}
$$

It is clear that $\tilde{Y}$ is non-negative. We look for a sub-solution for $(3.6)$ in the form $Q(x-c t)=$ $1-\beta e^{-\gamma(x-c t)}$, with $\beta, \gamma>0$. The function $Q(t, x, y)$ is a sub-solution for $\tilde{Y}$ if

$$
-\beta \gamma c e^{-\gamma \xi} \leq-\frac{\beta \gamma^{2}}{\mathrm{Le}} e^{-\gamma \xi}-M e^{-\lambda \xi}\left(1-\beta e^{-\gamma \xi}\right) .
$$

For this to hold when $\xi \geq 0$ it suffices that the following conditions are met: $0<\gamma \leq \lambda$ and

$$
-\beta \gamma c \leq-\frac{\beta \gamma^{2}}{\mathrm{Le}}-M
$$

They are satisfied if $\gamma>0$ is sufficiently small: $\gamma<c$ Le, and

$$
\beta \geq M\left(\gamma c-\frac{\gamma^{2}}{\mathrm{Le}}\right)^{-1} .
$$

In order for (3.7) to hold for $\xi<0$ it suffices to require

$$
-\beta \gamma c \leq-\frac{\beta \gamma^{2}}{\mathrm{Le}}+\beta M e^{-\lambda \xi}-M e^{-\lambda \xi+\gamma \xi} .
$$

This holds provided that $\beta \geq 1$ and $0<\gamma \leq c$ Le. Therefore, for $\beta>0$ large enough and $\gamma>0$ small enough, $R(x-c t)=\max \{0, Q(x-c t)\}$ provides a sub-solution for $\tilde{Y}(t, x, y)$ and hence for $Y(t, x, y)$, so that $Y(t, x, y) \geq R(x-c t)$ provided that

$$
Y_{0}(x, y) \geq 1-\beta e^{-\gamma x} .
$$


Note that both (3.5) and (3.9) hold if we choose $M$ and $\beta$ sufficiently large and $\gamma>0$ sufficiently small, because of our assumptions (3.2) on the rate at which $T_{0}$ and $Y_{0}$ approach zero and one, respectively.

Lastly, we observe that $T(t, x, y) \geq \tilde{T}(t, x, y)$, where

$$
\begin{aligned}
& \frac{\partial \tilde{T}}{\partial t}=\Delta \tilde{T}+R(x-c t) \tilde{T} \\
& \frac{\partial \tilde{T}}{\partial n}+q \tilde{T}=0, \quad y \in \partial \Omega \\
& \tilde{T}(0, x, y)=\tilde{T}_{0}(x, y) \leq T_{0}(x, y) .
\end{aligned}
$$

We look for $\tilde{T}(t, x, y)=g(x-c t) \phi(y)$, where $\phi$ is the eigenfunction defined by (3.3) and get an equation for the function $g(\xi)$ :

$$
-c g^{\prime}=g^{\prime \prime}-\mu g+R(\xi) g .
$$

It has two fundamental solutions $G_{1}$ and $G_{2}$, that are equal to $e^{\eta_{1,2} \xi}$ for $\xi<\gamma^{-1} \ln \beta$ (where $R(\xi)=0$ ), respectively, with $\eta_{1}>0$ and $\eta_{2}<0$ being the solutions of

$$
-c \eta=\eta^{2}-\mu .
$$

The function $G_{1}$ behaves as $e^{-\gamma x}$ as $x \rightarrow+\infty$, where $\gamma$ is a solution of

$$
-c \gamma=\gamma^{2}-\mu+1
$$

This equation has two solutions: $\gamma_{1}=\lambda$ and $\gamma_{2}>\lambda$ (recall that $\lambda$ is such that $\lambda<\sqrt{1-\mu}$ ). Therefore $G_{1}$ is a sub-solution for $T$ and, moreover, with an appropriate constant $B$ we have $T_{0}(x, y) \geq$ $B G_{1}(x) \phi(y)$. Therefore we have for all $t \geq 0$ :

$$
B G_{1}(x-c t) \phi(y) \leq T(t, x, y) \leq M e^{-\lambda(x-c t)}
$$

and hence $T$ moves with the speed $c$ in the sense of the theorem.

\subsection{Flame propagation in a shear flow}

Now we consider the KPP reaction-diffusion system (1.1) with a non-zero flow $u$ with the boundary conditions (1.3)

$$
\begin{aligned}
& \frac{\partial T}{\partial t}+u(y) T_{x}=\Delta T+T Y \\
& \frac{\partial Y}{\partial t}+u(y) Y_{x}=\frac{1}{\mathrm{Le}} \Delta Y-T Y .
\end{aligned}
$$

We have also set the amplitude $A=1$ to simplify the notation. The initial conditions $T_{0}$ and $Y_{0}$ are as in (3.2):

$$
\begin{aligned}
& 0 \leq T_{0}, \quad T_{0} \text { is bounded, } \quad 0 \leq Y_{0} \leq 1 \\
& C_{1} e^{-\lambda x} \leq T_{0}(x, y) \leq C_{2} e^{-\lambda x}, \quad x \geq 0, y \in \bar{\Omega} \\
& 1-Y_{0}(x, y) \leq C_{3} e^{-\lambda^{\prime} x}, \quad x \geq 0, y \in \bar{\Omega}, \quad \lambda^{\prime}>0
\end{aligned}
$$

for some positive constants $C_{1}, C_{2}, C_{3}$ and $\lambda^{\prime}$. In the sequel, we let $\phi_{\lambda}(y)$ be the positive eigenfunction of the eigenvalue problem (2.1) with the normalization (2.2) and with $s=\lambda$, while $\phi(y)=\phi_{0}(y)$ is the eigenfunction of (3.3), as in the previous section. 
We assume here, as we did in the uniform case, that $\mu(\lambda)<1$ to avoid the flame blow-off (at the fixed amplitude $A=1$ ). In this section, we will often suppress in notation the dependence of $\mu$ on $q$, as we just did above. We choose $c[\lambda]>0$ so that

$$
c \lambda-\lambda^{2}=1-\mu(\lambda) .
$$

We recall that $\mu(\lambda)$ has a variational representation (2.3) and is a concave function of $\lambda$ as a minimum of affine functions of $\lambda$. Therefore there exists a critical value $c_{*}$, so that (3.15), when interpreted as an equation for $\lambda$ with a given $c$, has no positive solutions for $c<c_{*}$, one positive solution $\lambda_{*}$ for $c=c_{*}$ and two positive solutions for $c>c_{*}$. We will assume that $\lambda<\lambda_{*}$. Note also that, as follows from $(2.6)$

$$
c^{*}>-\frac{d \mu}{d s}(0)=\int_{\Omega} u(y) \phi^{2}(y) d y,
$$

where $\phi$ is the first eigenfunction of (3.3), which does not depend on $u(y)$ and is normalized so that $\|\phi\|_{2}=1$.

We denote by $\mu^{N}(\lambda)$ the principal eigenvalue of the Neumann problem

$$
\begin{aligned}
& -\frac{1}{\operatorname{Le}} \Delta \Psi_{\lambda}-\lambda u(y) \Psi_{\lambda}=\mu^{N}(\lambda) \Psi_{\lambda}, \quad y \in \Omega \\
& \frac{\partial \Psi_{\lambda}}{\partial n}=0, \quad y \in \partial \Omega .
\end{aligned}
$$

Note that $\mu^{N}(\lambda) \leq 0$ since

$$
\mu^{N}(\lambda)=\min _{\psi \in H^{1}(\Omega),\|\psi\|_{L^{2}}=1}\left(\frac{1}{\operatorname{Le}} \int_{\Omega}|\nabla \psi(y)|^{2} d y-\lambda \int_{\Omega} u(y) \psi^{2}(y) d y\right) \leq 0,
$$

The inequality (3.17) is obtained using $\psi=|\Omega|^{-1 / 2}$ as a test function as well as taking the mean zero condition (1.4) into account. Furthermore, $\mu^{N}(\lambda)<0$ for all $\lambda \neq 0$ as soon as $u$ satisfies (1.4) and $u \not \equiv 0$.

We now prove the following theorem, which is an extension of Theorem 2 to the shear flow case.

Theorem 3 Assume that $\mu(0)<1, \mu(\lambda)<1$ and $\lambda<\lambda^{*}$. Let $c>0$ be defined by (3.15). The solution of (3.11-3.12) moves with the speed $c$ in the following sense. For any $c^{\prime}>c$ and any $(x, y) \in$ $\mathbb{R} \times \bar{\Omega}$ we have $T\left(t, x+c^{\prime} t, y\right) \rightarrow 0$ as $t \rightarrow+\infty$. On the other hand, there exists a nonnegative, not identically equal to zero function $G(x, y)$ such that if $T_{0}(x, y) \geq G(x, y)$, then $T(t, x+c t, y) \geq G(x, y)$ for all $t$.

Proof. Once again we will iteratively construct sub- and super-solutions for $T(t, x, y)$ that both move with the same speed $c[\lambda]$. As before, we have that $T(t, x, y) \geq 0$ and $0 \leq Y(t, x, y) \leq 1$ from the maximum principle so that the function $\bar{T}(t, x, y)=M e^{-\lambda(x-c t)} \phi_{\lambda}(y)$ satisfies the Robin boundary conditions and

$$
\frac{\partial \bar{T}}{\partial t}+u(y) \bar{T}_{x}=\Delta \bar{T}+\bar{T} \geq \Delta \bar{T}+\bar{T} Y
$$

for all $M$. Therefore, we have $T(t, x, y) \leq \bar{T}(t, x, y) \leq U(x-c t), U(\xi)=M\left\|\phi_{\lambda}\right\|_{\infty} e^{-\lambda \xi}$, for a sufficiently large $M$. This implies in particular that for $(x, y) \in \mathbb{R} \times \bar{\Omega}$ and $c^{\prime}>c$ we have

$$
T\left(t, x+c^{\prime} t, y\right) \leq M\left\|\phi_{\lambda}\right\|_{\infty} e^{-\lambda\left(x+c^{\prime} t-c t\right)} \rightarrow 0 \text { as } t \rightarrow+\infty
$$

and the first statement of the theorem follows. 
The previous argument implies that $Y(t, x, y) \geq \tilde{Y}(t, x, y) \geq 0$ with the function $\tilde{Y}$ that solves

$$
\begin{aligned}
& \frac{\partial \tilde{Y}}{\partial t}+u(y) \tilde{Y}_{x}=\frac{1}{\mathrm{Le}} \Delta \tilde{Y}-U(x-c t) \tilde{Y} \\
& \frac{\partial \tilde{Y}}{\partial n}=0, \quad y \in \partial \Omega \\
& \tilde{Y}(0, x, y)=Y_{0}(x, y) .
\end{aligned}
$$

We construct a sub-solution for (3.18) in the form $Q(x-c t, y)=1-\beta \Psi_{\gamma}(y) e^{-\gamma(x-c t)}$, where $\Psi_{\gamma}(y)$ is the eigenfunction of (3.16) with $\gamma$ sufficiently small to be determined. The function $Q(x-c t, y)$ is a sub-solution for $\tilde{Y}$ if

$$
(-\beta \gamma c+\gamma \beta u(y)) \Psi_{\gamma}(y) e^{-\gamma \xi} \leq-\frac{\beta \gamma^{2}}{\operatorname{Le}} \Psi_{\gamma}(y) e^{-\gamma \xi}-U(\xi)\left(1-\beta e^{-\gamma \xi} \Psi_{\gamma}(y)\right)-\frac{\beta}{\operatorname{Le}} e^{-\gamma \xi} \Delta \Psi_{\gamma}(y),
$$

or, equivalently,

$$
-\beta \gamma c-\mu^{N}(\gamma) \beta \leq-\frac{\beta \gamma^{2}}{\mathrm{Le}}-U(\xi) e^{\gamma \xi}\left(\frac{1}{\Psi_{\gamma}(y)}-\beta e^{-\gamma \xi}\right) .
$$

The above holds with $U(\xi)=M\left\|\phi_{\lambda}\right\|_{\infty} e^{-\lambda \xi}, 0<\gamma<\lambda$ and $\xi>0$, provided that

$$
-\beta \gamma c-\mu^{N}(\gamma) \beta \leq-\frac{\beta \gamma^{2}}{\mathrm{Le}}-\frac{M\left\|\phi_{\lambda}\right\|_{\infty}}{m_{\gamma}}
$$

where $m_{\gamma}=\inf _{y} \Psi_{\gamma}(y)>0$. Therefore, we may choose $\beta$ so that

$$
\beta \geq M\left\|\phi_{\lambda}\right\|_{\infty}\left(c \gamma-\frac{\gamma^{2}}{\mathrm{Le}}+\mu^{N}(\gamma)\right)^{-1} m_{\gamma}^{-1}
$$

Note that if we choose $\gamma$ sufficiently small, then $\beta>0$. Indeed, let $s(\gamma)=c \gamma-\frac{\gamma^{2}}{\mathrm{Le}}+\mu^{N}(\gamma)$. Then we have $s(0)=0$ and $s^{\prime}(0)=c+d \mu_{\gamma}^{N}(0) / d \gamma$. However, (2.6) taken with $q=0$, and $\Psi_{0}^{N}=1 /|\Omega|^{1 / 2}$, together with the mean-zero condition (1.4) on $u(y)$ show that $d \mu^{N}(\gamma) / d \gamma=0$ and hence the right side in (3.20) is positive for a sufficiently small $\gamma>0$. In order for (3.19) to hold when $\xi<0$, it is sufficient that

$$
-\beta \gamma c-\mu^{N}(\gamma) \beta \leq-\frac{\beta \gamma^{2}}{\mathrm{Le}}-M\left\|\phi_{\lambda}\right\|_{\infty} e^{-\lambda \xi+\gamma \xi}\left(\frac{1}{m_{\gamma}}-\beta e^{-\gamma \xi}\right) .
$$

However, with $\beta$ as in (3.20) condition (3.21) is satisfied if

$$
\frac{M\left\|\phi_{\lambda}\right\|_{\infty}}{m_{\gamma}} \geq M\left\|\phi_{\lambda}\right\|_{\infty} e^{-\lambda \xi+\gamma \xi}\left(\frac{1}{m_{\gamma}}-\beta e^{-\gamma \xi}\right)
$$

which is true if we take $M$ (and hence $\beta$ ) large enough so that the right side above is negative. Therefore $R(x-c t, y)=\max \{0, Q(x-c t, y)\}$ provides a sub-solution for $Y(t, x, y)$ and $Y(t, x, y) \geq$ $R(x-c t, y)$ provided that

$$
Y_{0}(x, y) \geq 1-\beta e^{-\gamma x} \Psi_{\gamma}(y) .
$$

This in turn implies that $Y(t, x, y) \geq \tilde{R}(x-c t)$, where $\tilde{R}(\xi)=\max \left\{0,1-\alpha e^{-\gamma \xi}\right\}$ with a sufficiently large $\alpha$ and a sufficiently small $\gamma$.

We look now for a sub-solution for $T$ in the form

$$
\underline{T}(t, x, y)=\phi_{\lambda}(y) e^{-\lambda(x-c t)}-k \phi_{\lambda+\eta}(y) e^{-(\lambda+\eta)(x-c t)}
$$


with the constant $k>0$ sufficiently large, and $0<\eta<\gamma$ sufficiently small. For the function $\underline{T}$ to be a sub-solution for $T$ in the region where $\alpha e^{-\gamma(x-c t)} \leq 1$, we should have

$$
\underline{T}_{t}+u(y) \underline{T}_{x} \leq \Delta \underline{T}+\left(1-\alpha e^{-\gamma(x-c t)}\right) \underline{T} .
$$

A direct calculation shows that this condition is satisfied provided that $\alpha>1$,

$$
p(\eta)=1-c(\eta+\lambda)-\mu(\eta+\lambda)+(\eta+\lambda)^{2}<0
$$

and $k$ is chosen so large that

$$
k>-\alpha m(\eta) / p(\eta)
$$

where $m(\eta)=\sup _{y}\left(\phi_{\lambda}(y) / \phi_{\eta+\lambda}(y)\right)$. Hence we need to find $0<\eta<\gamma$ so that $p(\eta)$ defined above is negative. One observes that $p(0)=0$, hence it is enough to prove that $p^{\prime}(0)<0$. We have

$$
p^{\prime}(0)=2 \lambda-c-\frac{d \mu}{d \lambda}(\lambda)
$$

and the function $\mu(\lambda)$ is concave (as has been shown in Section 2). Moreover, $\lambda$ is the smallest solution of the equation $\lambda^{2}+1=c \lambda+\mu(\lambda)$. Therefore we have $2 \lambda<c+\frac{d \mu}{d \lambda}(\lambda)$ and hence $p^{\prime}(0)$ is negative, so that there exists $0<\eta<\gamma$ so that $p(\eta)<0$.

Next we note that for $\underline{T}$ to be a sub-solution on the set $\alpha e^{-\gamma(x-c t)} \geq 1$ we should have

$$
\underline{T}_{t}+u(y) \underline{T}_{x} \leq \Delta \underline{T} .
$$

A similar calculation shows that a sufficient condition is

$$
k[-1+p(\eta)] \leq-m(\eta) e^{\eta x} .
$$

However, we have $e^{\eta x} \leq \alpha^{\eta / \gamma} \leq \alpha$ in this region provided that $\alpha>1$. This implies that (3.22) is sufficient for (3.23) to hold and hence $\underline{T}$ is a global sub-solution.

It follows in particular that there exists $x_{0} \in \mathbb{R}$ and $m(y)>0$ such that $T\left(t, x_{0}+c t, y\right) \geq m(y)$ for all $t>0$ and $y \in \Omega$. The last conclusion of Theorem 3 follows then from the a priori bounds for $T$ and $Y$ and from standard parabolic estimates and the strong parabolic maximum principle.

\section{Existence and qualitative properties of fronts}

This section is devoted to the problem of existence and qualitative properties of travelling fronts for the reaction-diffusion system (3.11) with the heat-loss boundary conditions (1.3). A travelling front is a pair of solutions of $(3.11)$ of the type $(T(x-c t, y), Y(x-c t, y))$ satisfying the asymptotic conditions

$$
\left\{\begin{array}{l}
T(+\infty, \cdot)=0, \quad Y(+\infty)=1 \\
T_{x}(-\infty, \cdot)=0, \quad Y_{x}(-\infty, \cdot)=0
\end{array}\right.
$$

together with the boundary conditions (1.3).

We pass to the moving frame coordinates: $x^{\prime}=x-c t$, and drop the primes to simplify the notation. Then a travelling front for $(3.11)$ is a pair $(T(x, y), Y(x, y))$ of functions solving

$$
\left\{\begin{array}{r}
\Delta T+(c-u(y)) T_{x}+T Y=0 \\
\frac{1}{\mathrm{Le}} \Delta Y+(c-u(y)) Y_{x}-T Y=0
\end{array} \text { in } \mathbb{R} \times \Omega\right.
$$

together with the boundary and asymptotic conditions above. The speed $c$ is an unknown of the problem. The limits in (4.1) are understood to be uniform with respect to $y \in \bar{\Omega}$. We also assume that 
$T$ and $Y$ are bounded, that $T \geq 0$ and $0 \leq Y \leq 1$, and that $(T, Y) \not \equiv(0,1)$ is not a trivial solution. The functions $T$ and $Y$ stand for the temperature and concentration profiles of the travelling front moving with speed $c$ to the right.

We recall that in the case of Le $=1$ and no heat-losses $(q=0)$, when the reaction-diffusion system (4.2) reduces to a single equation and $Y=1-T$, there exist traveling fronts that move with all speeds $c \geq c_{*}$ [8]. The speed $c$ is then related to the exponential decay of the traveling front by (3.15), with $\mu(\lambda)$ being the eigenvalue of (2.1) with $q=0$ that corresponds to the adiabatic case. The second part of the next theorem shows that this result may be extended to the heat-loss case and Le $=1$ with $\mu(\lambda)$ being the eigenvalue of $(2.1)$ with the heat-loss parameter $q \neq 0$. The first part of the theorem presents some necessary conditions for the existence of a front in the general case Le $\neq 1$, as well as some properties of such fronts. While these results are similar in spirit to those in $[4,8]$ obtained for a single reaction-diffusion equation in a shear flow, the method of the proof is based on the strategy developed in Section 3.

Theorem 4 a) If there exists a travelling front, namely a bounded solution $(c, T, Y)$ of (4.1-4.2) with the boundary conditions (1.3), then $T>0,0<Y<1, T(-\infty, \cdot)=0$ and $Y(-\infty, \cdot)$ is a constant $Y_{\infty}<1$. Furthermore, $\mu(0)<1$ (where $\mu(0)$ is the first eigenvalue of (2.1) with $s=0$ ), $c>0$ and $c \geq c^{*}$, where $c^{*}$ is the unique speed for which (3.15) has a single positive solution $\lambda^{*}$.

b) If Le $=1, \mu(0)<1$ and $c>\max \left(0, c^{*}\right)$, then there exists a bounded nonnegative solution $(T, Y)$ of (4.1-4.2) with the boundary conditions (1.3) that moves with the speed $c$.

We point out that, even if $L e=1$ in Part b) of Theorem 4, the system (4.2) does not reduce to a single equation. Part b) is then not a perturbative result and seems to be one of the first results on the existence of fronts for such a multidimensional thermo-diffusive system.

We comment on the relation between the necessary condition $c>0$ and the flame blow-off phenomenon described in Section 2 that seemingly corresponds to negative speeds. The restriction $c>0$ comes from the boundary condition $Y \rightarrow 1$ as $x \rightarrow+\infty$ which is incompatible with a nontrivial left-moving temperature front as the latter would have to consume some of the concentration on the right end of the domain. Left-moving fronts would have the boundary condition $Y \rightarrow 1$ as $x \rightarrow-\infty$.

Furthermore, we note that there is an interesting difference between the adiabatic and heat-loss cases even when Le $=1$. Theorem 4 implies the existence of traveling fronts for all $c>\max \left(0, c^{*}\right)$. In the adiabatic case $q=0$ we have $c^{*}>0$ and, moreover, $c^{*}$ itself is an admissible speed [8]. On the other hand, when the heat-loss parameter $q$ is positive, it is possible that equation (3.15) has two positive solutions $\lambda$ for $c=0$. Indeed, under the notation of Section 2 , let $\Omega, q_{0}>0$ and $u(y)$ be such that $\mu_{q_{0}}(0)=1$ and $d \mu_{q_{0}} / d s(0)>0$. Let $\lambda>0$ and $c_{0}<0$ be such that $c_{0} \lambda+\mu_{q_{0}}(\lambda)>1+\lambda^{2}$. By continuity with respect to $q$, there exists $q_{1} \leq q_{0}$ such that $\mu_{q_{1}}(0)<1$ and $c_{0} \lambda+\mu_{q_{1}}(\lambda)>1+\lambda^{2}$. Therefore, equation (3.15) with $c_{0}$ has at least one root, which implies that $c^{*}$ is negative. This implies that $c^{*}<0$ but $c=0$ is the infimum speed of propagation, but not a minimal speed. This corresponds to a blow-off situation.

The condition $\mu(0)<1$ is a necessary condition for the existence of fronts, and it is also sufficient for the existence of bounded fronts in the case Le $=1$. Note that this condition depends only on $q$ and on the domain $\Omega$, but not on the flow $u(y)$. However, the fastest possible decay rate $\lambda$ that is capable to keep the flame from being blown-off does depend on the flow $u(y)$. Furthermore, since $\mu(0)$ is less than the first eigenvalue $\mu^{D}$ of $-\Delta$ in $\Omega$ with Dirichlet boundary conditions on $\partial \Omega$, it follows that $\mu(0)<1$ if, for example, $\Omega$ contains a large ball, no matter how large $q$ is. On the other hand, when $\Omega$ is narrow enough so that $\mu^{D}>1$, then $\mu(0)>1$ for large heat loss rates $q$ and flame extinction occurs (see Theorem 1 ). This agrees qualitatively with the numerical observations in $[12,13]$. 
We also recall that $\mu(0)=\mu_{q}(0)$ is an increasing function of $q$. Since $\mu_{0}(0)=0<1$, fronts exist if and only if $q<q^{*}$, for some $0<q^{*} \leq+\infty$, while there are no fronts and quenching occurs for $q>q^{*}\left(\right.$ if $\left.q^{*}<+\infty\right)$.

Finally we comment on the case Le $\neq 1$. One may generalize Theorem 4 to this case as well constructing travelling fronts for $c>c^{*}$ (recall that $c^{*}$ is independent of the Lewis number). However, at the moment a uniform upper bound for $T$ in that case is not known so that one would have to deal solutions for $T$ that might grow exponentially as $x \rightarrow-\infty$.

Proof of part a). Let us assume here that $(c, T, Y)$ solves (4.1-4.2) with the boundary conditions (1.3). As already underlined, we assume that $T$ and $Y$ are bounded, $T \geq 0,0 \leq Y \leq 1$, and that $(T, Y) \not \equiv(0,1)$.

First, it follows from the maximum principle applied to $Y$ that $Y>0$. Let us then integrate the equation satisfied by $Y$ over a finite cylinder $\mathcal{C}_{N}=(-N, N) \times \Omega$. One gets

$$
\mathrm{Le}^{-1} \int_{\Omega}\left(Y_{x}(N, y)-Y_{x}(-N, y)\right) d y+\int_{\Omega}(c-u(y))(Y(N, y)-Y(-N, y)) d y-\int_{\mathcal{C}_{N}} T Y d x d y=0 .
$$

The first integral above goes to 0 as $N \rightarrow+\infty$, as $Y_{x}(-\infty, \cdot)=0$ by assumption while $Y_{x}(+\infty, \cdot)=0$ from $Y(+\infty, \cdot)=1$ and from standard elliptic estimates, as $T(+\infty, \cdot)=0$, and the second integral in (4.3) is bounded. Therefore, since $T Y$ is nonnegative, one concludes that the integral $\int_{\mathbb{R} \times \Omega} T Y d x d y$ converges.

We now multiply the equation for $Y$ by $Y$ and integrate by parts over $\mathcal{C}_{N}$. One gets

$$
\begin{aligned}
& \mathrm{Le}^{-1} \int_{\Omega}\left(Y Y_{x}(N, y)-Y Y_{x}(-N, y)\right) d y-\mathrm{Le}^{-1} \int_{\mathcal{C}_{N}}|\nabla Y|^{2} d x d y \\
& \quad+\frac{1}{2} \int_{\Omega}(c-u(y))\left(Y^{2}(N, y)-Y^{2}(-N, y)\right) d y-\int_{\mathcal{C}_{N}} T Y^{2} d x d y=0 .
\end{aligned}
$$

The first integral above goes to 0 as $N \rightarrow+\infty$, for the same reason as in (4.3), the third one is bounded and the fourth one converges (since $0 \leq T Y^{2} \leq T Y\|Y\|_{L^{\infty}}$ and $\int_{\mathbb{R} \times \Omega} T Y d x d y$ converges). Therefore, the integral $\int_{\mathbb{R} \times \Omega}|\nabla Y|^{2} d x d y$ converges.

Similarly, integrating the equation for $T$ yields the convergence of $\int_{\mathbb{R} \times \partial \Omega} T d x d y$. Furthermore, multiplying the equation for $T$ by $T$ and integrating by parts leads to the convergence of $\int_{\mathbb{R} \times \Omega}|\nabla T|^{2} d x d y$.

For each $n \in \mathbb{N}$ we denote the shifted functions $T_{n}(x, y)=T(x-n, y)$ and $Y_{n}(x, y)=Y(x-n, y)$, and recall that the functions $T$ and $Y$ are globally bounded. Since $\left(T_{n}, Y_{n}\right)$ satisfy the same equations as $(T, Y)$, standard elliptic estimates imply that, along a subsequence $n^{\prime} \rightarrow+\infty,\left(T_{n^{\prime}}, Y_{n^{\prime}}\right) \rightarrow$ $\left(T_{\infty}, Y_{\infty}\right)$ in, at least, $C_{l o c}^{1}(\mathbb{R} \times \bar{\Omega})$ as $n^{\prime} \rightarrow+\infty$. Moreover, since $\int_{\mathbb{R} \times \Omega}|\nabla T|^{2}+|\nabla Y|^{2}<+\infty$, it follows that $T_{\infty}$ and $Y_{\infty}$ are two constants. Since $\frac{\partial T_{\infty}}{\partial n}+q T_{\infty}=0$ on $\mathbb{R} \times \partial \Omega$, one has $T_{\infty}=0$. Notice that $T_{\infty}=0$ does not depend on any subsequence, whence $T(x, y) \rightarrow 0$ as $x \rightarrow-\infty$ uniformly in $y \in \bar{\Omega}$.

Taking $N=n^{\prime}$ in (4.3) and passing to the limit $n^{\prime} \rightarrow+\infty$ leads to

$$
c\left(1-Y_{\infty}\right)|\Omega|=\int_{\mathbb{R} \times \Omega} T Y d x d y
$$

where $|\Omega|$ denotes the Lebesgue measure of $\Omega$. One here uses (1.4), and the fact that $Y_{x}\left( \pm n^{\prime}, y\right) \rightarrow 0$ uniformly in $y$.

If $T$ were identically equal to 0 , then $c\left(1-Y_{\infty}\right)=0$. On the other hand, it would then follow from (4.4), by passing to the limit $N=n^{\prime} \rightarrow+\infty$, that $\operatorname{Le}^{-1} \int_{\mathbb{R} \times \Omega}|\nabla Y|^{2} d x d y=c\left(1-Y_{\infty}^{2}\right)|\Omega| / 2=0$, whence $Y \equiv 1$ from (4.1). 
Therefore, $T \not \equiv 0$, whence $T>0$ from the strong maximum principle. Hence, it follows from (4.5) that $c\left(1-Y_{\infty}\right)>0$ and that $Y_{\infty}$ does not depend on any subsequence. As a consequence, we conclude that $Y(x, y) \rightarrow Y_{\infty} \neq 1$ as $x \rightarrow-\infty$ uniformly in $y \in \bar{\Omega}$. Furthermore, since $Y \leq 1$, we have $Y_{\infty}<1$ and thus $c>0$. The strong maximum principle then yields $Y<1$ in $\mathbb{R} \times \bar{\Omega}$.

Let us now prove that $\mu(0)<1$, where $\mu(0)$ is the first eigenvalue of $(2.1)$ with $s=0$. Assume by contradiction that $\mu(0) \geq 1$. The first eigenfunction $\phi=\phi_{0}$ of $(2.1)$ with $s=0$ ( $\phi$ is the first eigenfunction of (3.3)) satisfies in that case

$$
\left\{\begin{aligned}
\Delta \phi+\phi & \leq 0 \text { in } \Omega \\
\frac{\partial \phi}{\partial n}+q \phi & =0 \text { on } \partial \Omega
\end{aligned}\right.
$$

and $\phi>0$ in $\bar{\Omega}$. Since $T$ is bounded, one then has $T(x, y) \leq \gamma \phi(y)$ in $\mathbb{R} \times \bar{\Omega}$ for all $\gamma>0$ large enough. Since $T>0$ and $T( \pm \infty, \cdot)=0$, there exists then $\gamma^{*}>0$ such that $T(x, y) \leq \gamma^{*} \phi(y)$ in $\mathbb{R} \times \bar{\Omega}$ with equality somewhere. But since $T \geq 0$ and $Y \leq 1$, the function $T$ satisfies

$$
\Delta T+(c-u(y)) T_{x}+T \geq \Delta T+(c-u(y)) T_{x}+T Y=0 \text { in } \mathbb{R} \times \Omega .
$$

Therefore, $z(x, y)=T(x, y)-\gamma^{*} \phi(y)$ is non-positive in $\mathbb{R} \times \bar{\Omega}$, vanishes somewhere, and satisfies

$$
\Delta z+(c-u(y)) z_{x}+z \geq 0 \text { in } \mathbb{R} \times \Omega
$$

together with the Robin boundary conditions $\frac{\partial z}{\partial n}+q z=0$ on $\mathbb{R} \times \partial \Omega$. It follows then from the strong maximum principle and Hopf lemma that $z \equiv 0$, whence $T(x, y) \equiv \gamma^{*} \phi(y)$. The latter is impossible since, say, $T( \pm \infty, \cdot)=0$ and $\gamma^{*}>0$. This contradiction shows that $\mu(0)<1$.

As noticed at the beginning of Section 3.2, there exists then $c^{*} \in \mathbb{R}$ which is the unique speed for which (3.15) has a single positive solution $\lambda^{*}$. Let us now prove that $c \geq c^{*}$. We first observe that, since both $T$ and $Y$ are positive, bounded, and satisfy (4.2), it follows from the standard elliptic estimates and the Harnack inequalities up to the boundary that the function $|\nabla T| / T$ is globally bounded in $\mathbb{R} \times \bar{\Omega}$. Let us introduce

$$
\lambda=-\liminf _{x \rightarrow+\infty, y \in \bar{\Omega}} \frac{T_{x}(x, y)}{T(x, y)},
$$

the "exponential rate" of decay of $T$ to zero at infinity. Since $T(+\infty, \cdot)=0$ and $T>0$ in $\mathbb{R} \times \Omega$, one has $\lambda \geq 0$. Let $\left(x_{k}, y_{k}\right) \in \mathbb{R} \times \bar{\Omega}$ be a sequence such that $x_{k} \rightarrow+\infty$ and

$$
\frac{T_{x}\left(x_{k}, y_{k}\right)}{T\left(x_{k}, y_{k}\right)} \rightarrow-\lambda \text { as } k \rightarrow+\infty
$$

We denote $\tilde{T}_{k}(x, y)=T\left(x+x_{k}, y\right) / T\left(x_{k}, y_{k}\right)$. The functions $\tilde{T}_{k}$ are locally bounded and satisfy

$$
\left\{\begin{aligned}
\Delta \tilde{T}_{k}+(c-u(y))\left(\tilde{T}_{k}\right)_{x}+\tilde{T}_{k}(x, y) Y\left(x+x_{k}, y\right) & =0 \text { in } \mathbb{R} \times \Omega \\
\frac{\partial \tilde{T}_{k}}{\partial n}+q \tilde{T}_{k} & =0 \text { on } \mathbb{R} \times \partial \Omega .
\end{aligned}\right.
$$

On the other hand, the function $Y$ is bounded and $Y(+\infty, \cdot)=1$. It follows then from the standard elliptic estimates up to the boundary that the functions $\tilde{T}_{k^{\prime}}$ converge along a subsequence $k^{\prime} \rightarrow+\infty$ weakly in $W_{l o c}^{2, p}$ for all $p>1$ and strongly in $C_{l o c}^{1, \alpha}$ for all $0 \leq \alpha<1$, to a function $\tilde{T}_{\infty}$ solving

$$
\left\{\begin{aligned}
\Delta \tilde{T}_{\infty}+(c-u(y))\left(\tilde{T}_{\infty}\right)_{x}+\tilde{T}_{\infty} & =0 \text { in } \mathbb{R} \times \Omega \\
\frac{\partial \tilde{T}_{\infty}}{\partial n}+q \tilde{T}_{\infty} & =0 \text { on } \mathbb{R} \times \partial \Omega
\end{aligned}\right.
$$


Up to extraction of another subsequence, one can assume that $y_{k^{\prime}} \rightarrow y_{\infty} \in \bar{\Omega}$. Therefore, we have $\tilde{T}_{\infty}\left(0, y_{\infty}\right)=1$, and the nonnegative function $\tilde{T}_{\infty}$ is positive in $\mathbb{R} \times \bar{\Omega}$ because of the strong maximum principle.

On the other hand, it follows from the definitions of $\lambda$ and of the sequence $\left(x_{k}, y_{k}\right)$ that

$$
\frac{\left(\tilde{T}_{\infty}\right)_{x}}{\tilde{T}_{\infty}} \geq-\lambda \text { in } \mathbb{R} \times \bar{\Omega} \text { and } \frac{\left(\tilde{T}_{\infty}\right)_{x}\left(0, y_{\infty}\right)}{\tilde{T}_{\infty}\left(0, y_{\infty}\right)}=-\lambda
$$

Therefore, the function $z=\left(\tilde{T}_{\infty}\right)_{x} / \tilde{T}_{\infty}$ is such that $z \geq-\lambda, z\left(0, y_{\infty}\right)=-\lambda$, and it satisfies

$$
\left\{\begin{aligned}
\Delta z+\frac{2 \nabla \tilde{T}_{\infty}}{\tilde{T}_{\infty}} \cdot \nabla z+(c-u(y)) z_{x} & =0 \text { in } \mathbb{R} \times \Omega \\
\frac{\partial z}{\partial n} & =0 \text { on } \mathbb{R} \times \partial \Omega .
\end{aligned}\right.
$$

It then follows from the strong maximum principle and Hopf lemma that $z \equiv-\lambda$. In other words, the positive function $\tilde{T}_{\infty}$ can be written as $\tilde{T}_{\infty}(x, y)=e^{-\lambda x} \varphi(y)$, where the positive function $\varphi$ satisfies

$$
\left\{\begin{aligned}
\Delta \varphi-\lambda(c-u(y)) \varphi+\left(\lambda^{2}+1\right) \varphi & =0 \text { in } \Omega \\
\frac{\partial \varphi}{\partial n}+q \varphi & =0 \text { on } \partial \Omega .
\end{aligned}\right.
$$

By uniqueness of the positive eigenfunction, one concludes that $\varphi$ is the first eigenfunction (up to multiplication) for problem (2.1) with $s=\lambda$, and that (3.15) is satisfied, namely $c \lambda-\lambda^{2}=1-\mu(\lambda)$. Since $\lambda \geq 0$ and the last equation cannot be satisfied for $\lambda=0$ (since $\mu(0)<1$ ), one obtains $\lambda>0$.

From the characterization of $c^{*}$, one concludes then that $c \geq c^{*}$. That completes the proof of part a) of Theorem 4.

Proof of part b). We assume that $\mathrm{Le}=1, \mu(0)<1$ and fix $c>\max \left(0, c^{*}\right)$. The proof of the existence of a nonnegative solution $(T, Y)$ of (4.1-4.2) satisfying the boundary conditions (1.3) is based on the choice of suitable sub- and super-solutions for both $T$ and $Y$ (from Section 3.2), and on the construction of approximate solutions in bounded cylinders (the latter step is similar to the methods that were used, for example, in $[5,8]$ ) with the subsequent passage to the limit of an infinite cylinder.

Let $\lambda$ be the smallest positive solution of (3.15), and let

$$
\bar{T}(x, y):=\phi_{\lambda}(y) e^{-\lambda x},
$$

where $\phi_{\lambda}$ is the first eigenfunction of (2.1) with $s=\lambda$. The function $\bar{T}$ satisfies

$$
\left\{\begin{aligned}
\Delta \bar{T}+(c-u(y)) \bar{T}_{x}+\bar{T} & =0 \text { in } \mathbb{R} \times \Omega \\
\frac{\partial \bar{T}}{\partial n}+q \bar{T} & =0 \text { on } \mathbb{R} \times \partial \Omega .
\end{aligned}\right.
$$

On the other hand, it follows from the calculations in Section 3.2 that the function

$$
\underline{Y}(x, y):=\max \left(0,1-\beta \Psi_{\gamma}(y) e^{-\gamma x}\right)
$$

is a sub-solution of

$$
\left\{\begin{aligned}
\Delta \underline{Y}+(c-u(y)) \underline{Y}_{x}-\bar{T} \underline{Y} & \geq 0 \text { in } \mathbb{R} \times \Omega \\
\frac{\partial \underline{Y}}{\partial n} & =0 \text { on } \mathbb{R} \times \partial \Omega,
\end{aligned}\right.
$$

in the sense of distributions, for $\beta>0$ large enough and for $\gamma \in(0, \lambda)$ small enough. Here $\Psi_{\gamma}$ is the first eigenfunction of problem (3.16) with parameter $\lambda=\gamma$ and $\mathrm{Le}=1$. 
Finally, the calculations in Section 3.2 imply that the function

$$
\underline{T}(x, y):=\max \left(0, \phi_{\lambda}(y) e^{-\lambda x}-\kappa \phi_{\lambda+\eta}(y) e^{-(\lambda+\eta) x}\right)=\max \left(0, \bar{T}(x, y)-\kappa \phi_{\lambda+\eta}(y) e^{-(\lambda+\eta) x}\right)
$$

satisfies

$$
\left\{\begin{aligned}
\Delta \underline{T}+(c-u(y)) \underline{T}_{x}+\underline{Y} \underline{T} & \geq 0 \text { in } \mathbb{R} \times \Omega \\
\frac{\partial \underline{T}}{\partial n}+q \underline{T} & =0 \text { on } \mathbb{R} \times \partial \Omega,
\end{aligned}\right.
$$

in the sense of distributions, for $0<\eta<\gamma$ small enough and $\kappa>0$ large enough.

Lemma 1 There exists $a_{0}>0$ such that, for all $a>a_{0}$, there exists a classical solution $(T, Y)$ of

$$
\left\{\begin{array}{l}
\Delta T+(c-u(y)) T_{x}+T Y=0 \\
\Delta Y+(c-u(y)) Y_{x}-T Y=0
\end{array} \text { in } \Sigma_{a}=(-a, a) \times \Omega\right.
$$

and

$$
\left\{\begin{aligned}
\frac{\partial T}{\partial n}+q T & =0 \text { on }(-a, a) \times \partial \Omega \\
\frac{\partial Y}{\partial n} & =0 \text { on }(-a, a) \times \partial \Omega \\
T( \pm a, y)=\underline{T}( \pm a, y), \quad Y( \pm a, y) & =\underline{Y}( \pm a, y), y \in \Omega
\end{aligned}\right.
$$

such that $T$ and $Y$ are nonnegative, continuous in $\overline{\Sigma_{a}}$ and satisfy

$$
\underline{T} \leq T \leq \bar{T}, \underline{Y} \leq Y \leq 1 \text { in } \overline{\Sigma_{a}}
$$

together with

$$
0 \leq T+Y \leq 1 \text { in } \overline{\Sigma_{a}} .
$$

Proof. Let us first fix $a>a_{0}$ large enough so that

$$
\underline{T}(a, y)+\underline{Y}(a, y) \leq 1 \text { for all } y \in \bar{\Omega} .
$$

This is possible, owing to the definitions of $\underline{T}$ and $\underline{Y}$, since $0<\gamma<\lambda$. Without loss of generality, one can also assume that $\underline{T}(-a, y)=\underline{Y}(-a, y)=0$ and that $\underline{Y}(a, y)=1-\beta \Psi_{\gamma}(y) e^{-\gamma a}, \underline{T}(a, y)=$ $\bar{T}(a, y)-\kappa \phi_{\lambda+\eta}(y) e^{-(\lambda+\eta) a}$ for all $y \in \bar{\Omega}$.

Let $C\left(\overline{\Sigma_{a}}\right)$ denote the Banach space of continuous functions in $\overline{\Sigma_{a}}$, with the usual sup-norm. Observe that $0 \leq \underline{T}<\bar{T}$ and $0 \leq \underline{Y}<1$ in $\mathbb{R} \times \bar{\Omega}$ and denote by $E$ the set

$$
E=\left\{(T, Y) \in C\left(\overline{\Sigma_{a}}\right) \times C\left(\overline{\Sigma_{a}}\right), \underline{T} \leq T \leq \bar{T} \text { and } \underline{Y} \leq Y \leq 1 \text { in } \overline{\Sigma_{a}}\right\} .
$$

The set $E$ is a convex closed bounded subset of the Banach space $C\left(\overline{\Sigma_{a}}\right) \times C\left(\overline{\Sigma_{a}}\right)$. For any $\left(T_{0}, Y_{0}\right) \in$ $E$, let $(T, Y)=\Phi\left(T_{0}, Y_{0}\right)$ be the unique solution of

$$
\left\{\begin{aligned}
\Delta T+(c-u(y)) T_{x} & =-T_{0} Y_{0} & & \text { in } \Sigma_{a} \\
\Delta Y+(c-u(y)) Y_{x}-T_{0} Y & =0 & & \text { in } \Sigma_{a}
\end{aligned}\right.
$$

with the boundary conditions (4.7). Such a solution $(T, Y)$ exists, belongs to $C\left(\overline{\Sigma_{a}}\right) \times C\left(\overline{\Sigma_{a}}\right)$ (see $[5,8]$ for more details on similar problems) and is unique as follows from the maximum principle. Furthermore, it follows from the definitions of $E$ and $\underline{T}, \bar{T}$ and $\underline{Y}$, that

$$
\underline{T} \leq T \leq \bar{T} \text { and } \underline{Y} \leq Y \leq 1 \text { in } \overline{\Sigma_{a}} .
$$

Let us check for instance the first inequality : the function $\underline{T}$ satisfies $\Delta \underline{T}+(c-u(y)) \underline{T}_{x} \geq-\underline{Y} \underline{T} \geq$ $-T_{0} Y_{0}$ in the sense of distributions in $\Sigma_{a}$. Furthermore, $\underline{T}$ obviously satisfies the boundary conditions 
(4.7) for $T$. Therefore, $\underline{T} \leq T$ in $\overline{\Sigma_{a}}$. The other inequalities $T \leq \bar{T}, \underline{Y} \leq Y$ and $Y \leq 1$ can be checked similarly. Therefore, one concludes that $\Phi(E) \subset E$.

Furthermore, let us denote $\left(h_{1}, k_{1}\right)=\Phi(\bar{T}, 1)$ and $\left(h_{2}, k_{2}\right)=\Phi(\underline{T}, 1)$. For any $\left(T_{0}, Y_{0}\right) \in E$ and $(T, Y)=\Phi\left(T_{0}, Y_{0}\right)$, one has

$$
\Delta h_{1}+(c-u(y))\left(h_{1}\right)_{x}=-\bar{T} \leq-T_{0} Y_{0} \text { in } \Sigma_{a}
$$

whence $T \leq h_{1}$ in $\overline{\Sigma_{a}}$ (recall that $h_{1}$ satisfies the same boundary conditions $(4.7)$ as $T$ ). Similarly, one checks that

$$
\Delta k_{2}+(c-u(y))\left(k_{2}\right)_{x}-T_{0} k_{2}=\left(\underline{T}-T_{0}\right) k_{2} \leq 0 \text { in } \Sigma_{a},
$$

whence $Y \leq k_{2}$ in $\overline{\Sigma_{a}}$. Thus we obtain

$$
\underline{T} \leq T \leq h_{1} \leq \bar{T} \text { and } \underline{Y} \leq Y \leq k_{2} \leq 1 \text { in } \overline{\Sigma_{a}}
$$

for all $\left(T_{0}, Y_{0}\right) \in E$ and $(T, Y)=\Phi\left(T_{0}, Y_{0}\right)$.

Let now $\left(T_{0}^{n}, Y_{0}^{n}\right)_{n \in \mathbb{N}}$ be a sequence in $E$, and let $\left(T^{n}, Y^{n}\right)=\Phi\left(T_{0}^{n}, Y_{0}^{n}\right)$. As follows from the standard elliptic estimates up to the boundary, the sequence $\left(T^{n}, Y^{n}\right)$ is bounded in $C^{1}(K)$ norm, for any compact subset $K \subset \overline{\Sigma_{a}} \backslash\{ \pm a\} \times \partial \Omega$. Therefore, using the diagonal extraction process, we conclude that there exists a subsequence, still denoted by $\left(T^{n}, Y^{n}\right)$, which converges locally uniformly in $\overline{\Sigma_{a}} \backslash\{ \pm a\} \times \partial \Omega$ to a pair $(T, Y)$ of continuous functions in $\overline{\Sigma_{a}} \backslash\{ \pm a\} \times \partial \Omega$. Since each $\left(T^{n}, Y^{n}\right)$ satisfies (4.10) in $\overline{\Sigma_{a}}$, it follows that $(T, Y)$ satisfies (4.10) in $\overline{\Sigma_{a}} \backslash\{ \pm a\} \times \partial \Omega$. Since $h_{1}( \pm a, \cdot)=\underline{T}( \pm a, \cdot), k_{2}( \pm a, \cdot)=\underline{Y}( \pm a, \cdot)$, and both $\underline{T}, \underline{Y}, h_{1}$ and $k_{2}$ are continuous in $\overline{\Sigma_{a}}$, the functions $(T, Y)$ can be extended in $\overline{\Sigma_{a}}$ to two continuous functions, still denoted by $(T, Y)$, satisfying (4.10) in $\overline{\Sigma_{a}}$. For any $\varepsilon>0$, there exists $\delta>0$ such that

$$
0 \leq h_{1}-\underline{T} \leq \varepsilon \text { and } 0 \leq k_{2}-\underline{Y} \leq \varepsilon \text { in }[-a,-a+\delta] \times \bar{\Omega} \cup[a-\delta, a] \times \bar{\Omega},
$$

whence $\left|T^{n}-T\right| \leq \varepsilon$ and $\left|Y^{n}-Y\right| \leq \varepsilon$ in the same sets, for all $n$. On the other hand, $\left(T^{n}, Y^{n}\right)$ converges uniformly in $[-a+\delta, a-\delta] \times \bar{\Omega}$ to $(T, Y)$. Therefore, $\left(T^{n}, Y^{n}\right)$ converges uniformly to $(T, Y)$ in $\overline{\Sigma_{a}}$.

As a consequence, the set $\overline{\Phi(E)}$ is compact in $E$. One concludes from the Schauder fixed point theorem that $\Phi$ has a fixed point in $E$. Therefore, there exists a classical solution $(T, Y)=\left(T_{a}, Y_{a}\right)$ of problem (4.6) satisfying (4.7-4.8). Note that in particular $T$ and $Y$ are nonnegative.

Finally, the continuous function $Z=T+Y$ satisfies $\Delta Z+(c-u(y)) Z_{x}=0$ in $\Sigma_{a}$, together with the boundary condition

$$
\frac{\partial Z}{\partial n}=-q T \leq 0 \text { on }(-a, a) \times \partial \Omega
$$

and $Z(-a, y)=0, Z(a, y) \leq 1$ from (4.9). Therefore, $Z \leq 1$ in $\overline{\Sigma_{a}}$ and the proof of Lemma 1 is complete.

Let $\left(a_{n}\right)_{n \in \mathbb{N}^{*}}$ be an increasing sequence such that $a_{n}>a_{0}$ for all $n \geq 1$. From Lemma 1 , there exists, for each $n$, a classical solution $\left(T_{n}, Y_{n}\right)$ of (4.6-4.8). Furthermore, $T_{n}+Y_{n} \leq 1$, whence $T_{n} \leq 1$ in $\overline{\Sigma_{a_{n}}}$ (remember that $\left.Y_{n} \geq 0\right)$. Therefore, the functions $T_{n}$ and $Y_{n}$ are uniformly bounded between 0 and 1. From the standard elliptic estimates, the functions $\left(T_{n}, Y_{n}\right)$ are then locally bounded in, say, $C^{2, \alpha}$ for all $0 \leq \alpha<1$. Up to extraction of some subsequence, the functions $\left(T_{n}, Y_{n}\right)$ converge locally to two $C^{2}(\mathbb{R} \times \bar{\Omega})$ nonnegative solutions $(T, Y)$ of

$$
\left\{\begin{array}{l}
\Delta T+(c-u(y)) T_{x}+T Y=0 \\
\Delta Y+(c-u(y)) Y_{x}-T Y=0
\end{array} \text { in } \mathbb{R} \times \Omega\right.
$$


with the boundary conditions

$$
\frac{\partial T}{\partial n}+q T=0, \quad \frac{\partial Y}{\partial n}=0, \quad y \in \partial \Omega
$$

In other words, $(T, Y)$ solves (4.2) (with Le $=1$ ) and (1.3). Furthermore, $0 \leq \underline{T} \leq T \leq \min (\bar{T}, 1)$ and $0 \leq \underline{Y} \leq Y \leq 1$. Hence, $T(+\infty, y)=0$ and $Y(+\infty, y)=1$ uniformly in $y \in \bar{\Omega}$.

It only remains to check the limiting conditions (4.1) at $-\infty$. Let us first notice that $|\nabla T|$ and $|\nabla Y|$ are globally bounded in $\mathbb{R} \times \Omega$, from the standard elliptic estimates. Therefore, like in the beginning of the proof of part a) of Theorem 4, it follows from $(4.3)$ (with Le $=1$ ) that the integral $\int_{\mathbb{R} \times \Omega} T Y$ converges (absolutely). From (4.4) (with Le $=1$ ), one deduces that the integral $\int_{\mathbb{R} \times \Omega}|\nabla Y|^{2}$ converges as well. Similarly, one gets the convergence of $\int_{\mathbb{R} \times \Omega}|\nabla T|^{2}$.

Furthermore, as in part a), there exists a sequence of integers $m \rightarrow+\infty$ such that the functions $T_{m}(x, y)=T(x-m, y)$ and $Y_{m}(x, y)=Y(x-m, y)$ converge, at least in $C_{l o c}^{1}$, to two constants $T_{\infty}=0$ and $Y_{\infty}$. Since $T_{\infty}$ does not depend on the sequence $(m)$, one concludes that $T(x, y) \rightarrow 0$ and $|\nabla T(x, y)| \rightarrow 0$ as $x \rightarrow-\infty$ uniformly in $y \in \bar{\Omega}$. Notice that $|\nabla Y(x-m, y)| \rightarrow 0$ as $m \rightarrow+\infty$ locally in $(x, y) \in \mathbb{R} \times \bar{\Omega}$, and that $|\nabla Y(x, y)| \rightarrow 0$ as $x \rightarrow+\infty$ uniformly in $y \in \bar{\Omega}$ (with the same type of arguments). Therefore, putting $N=m$ in (4.3) (with Le $=1$ ) implies that

$$
c\left(1-Y_{\infty}\right)|\Omega|=\int_{\mathbb{R} \times \Omega} T Y d x d y .
$$

But since $\underline{T} \leq T$, one has $T \geq 0, \not \equiv 0$, whence $T>0$ from the strong maximum principle. It also follows that $Y \not \equiv 1$, whence $Y<1$ from the strong maximum principle. Furthermore, $Y(+\infty, \cdot)=1$, whence $Y>0$ in $\mathbb{R} \times \bar{\Omega}$. Therefore, the integral $\int_{\mathbb{R} \times \Omega} T Y$ is positive. Hence, $c>0, Y_{\infty}<1$ and $Y_{\infty}$ does not depend on the sequence $(m)$. As a consequence, $Y(x, y) \rightarrow Y_{\infty}$ and $|\nabla Y(x, y)| \rightarrow 0$ as $x \rightarrow-\infty$, uniformly in $y \in \bar{\Omega}$. That completes the proof of Theorem 4 .

\section{References}

[1] M. Abel, A. Celani, D. Vergni and A. Vulpiani, Front propagation in laminar flows, Physical Review E, 64, 2001, 046307.

[2] M. Abel, M. Cencini, D. Vergni and A. Vulpiani, Front speed enhancement in cellular flows, Chaos 12, 2003, 481.

[3] B. Audoly, H. Berestycki and Y. Pomeau, Réaction-diffusion en écoulement stationnaire rapide, C. R. Acad. Sci. Paris, 328 II, 2000, 255-262.

[4] H. Berestycki, The influence of advection on the propagation of fronts in reaction-diffusion equations, in Nonlinear PDEs in Condensed Matter and Reactive Flows, NATO Science Series C, 569, H. Berestycki and Y. Pomeau eds, Kluwer, Doordrecht, 2003.

[5] H. Berestycki and F. Hamel, Front propagation in periodic excitable media, Comm. Pure Appl. Math. 55, 2002, 949-1032.

[6] H. Berestycki, B. Larrouturou and P.-L. Lions, Multi-dimensional traveling wave solutions of a flame propagation model, Arch. Rational Mech. Anal., 111, 1990, 33-49.

[7] H. Berestycki, B. Larrouturou, P.-L. Lions and J.-M. Roquejoffre, An elliptic system modelling the propagation of a multidimensional flame, Unpublished manuscript. 
[8] H. Berestycki and L. Nirenberg, Traveling fronts in cylinders, Annales de l'IHP, Analyse non linéare, 9, 1992, 497-572.

[9] J. Billingham and D. Needham, The development of traveling waves in a quadratic and cubic autocatalysis with unequal diffusion. I. Permanent form traveling waves, Phil. Trans. R. Soc. Lond. A 334, 1991, 1-24.

[10] P. Constantin, A. Kiselev, A. Oberman and L. Ryzhik, Bulk burning rate in passive-reactive diffusion, Arch. Rat. Mech. Anal. 154, 2000, 53-91.

[11] P. Constantin, A. Kiselev and L. Ryzhik, Quenching of flames by fluid advection, Comm. Pure Appl. Math 54, 2001, 1320-1342.

[12] J. Daou and M. Matalon, Flame propagation in Poiseuille flow under adiabatic conditions, Combustion and Flame, 124, 2001, 337-349.

[13] J. Daou and M. Matalon, Influence of conductive heat-losses on the propagation of premixed flames in channels, Combustion and Flame 128, 2001, 321-339.

[14] S. Heinze, G. Papanicolaou and A. Stevens, Variational principles for propagation speeds in inhomogeneous media, SIAM J. Appl. Math. 62, 2001, 129-148.

[15] B. Khoudier, A. Bourlioux and A. Majda, Parametrizing the burning rate speed enhancement by small scale periodic flows: I. Unsteady shears, flame residence time and bending," Combust. Theory Model., 5, 2001, 295-318.

[16] L. Kagan and G. Sivashinsky, Flame propagation and extinction in large-scale vortical flows, Combust. Flame 120, 2000, 222-232.

[17] L. Kagan, P.D. Ronney and G. Sivashinsky, Activation energy effect on flame propagation in large-scale vortical flows, Combust. Theory Modelling 6, 2002, 479-485.

[18] A. Kiselev and L. Ryzhik, Enhancement of the travelling front speeds in reaction-diffusion equations with advection, Ann. Inst. H. Poincaré Anal. Non Linéaire 18, 2001, 309-358.

[19] A. Kiselev and L. Ryzhik, An upper bound for the bulk burning rate for systems, Nonlinearity, 14, 2001, 1297-1310.

[20] J.-F. Mallordy and J.-M. Roquejoffre, A parabolic equation of the KPP type in higher dimensions, SIAM J. Math. Anal., 26, 1995, 1-20.

[21] J. Nolen and J. Xin, Reaction-diffusion front speeds in spatially-temporally periodic shear flows, SIAM Jour. MMS, 1, 2003, 554-570.

[22] N. Peters, Turbulent combustion, Cambridge University Press, 2000.

[23] R. Texier-Picard and V. Volpert, Problèmes de réaction-diffusion-convection dans des cylindres non bornés, C. R. Acad. Sci. Paris Sr. I Math. 333, 2001, 1077-1082

[24] N. Vladimirova, P. Constantin, A. Kiselev, O. Ruchayskiy and L. Ryzhik, Flame Enhancement and Quenching in Fluid Flows, Combust. Theory Model., 7, 2003, 487-508

[25] V.A. Volpert and A.I. Volpert, Existence and stability of multidimensional travelling waves in the monostable case, Israel Jour. Math., 110, 1999, 269-292. 
[26] J. Xin, Existence of planar flame fronts in convective-diffusive periodic media, Arch. Rat. Mech. Anal., 121, 1992, 205-233.

[27] J. Xin, Existence and nonexistence of travelling waves and reaction-diffusion front propagation in periodic media, Jour. Stat. Phys., 73, 1993, 893-926.

[28] J. Xin, Analysis and modelling of front propagation in heterogeneous media, SIAM Rev., 42, 2000, 161-230.

[29] J. Xin, KPP front speeds in random shears and the parabolic Anderson model, to appear in Meth. Appl. Anal., 2003. 